\title{
Lipemia pós-prandial e incretinas na reatividade endotelial
}

\author{
Postprandial lipemia and incretins in endothelial reactivity
}

Priscila A. Maranhão*, Eliete Bouskela, Luiz Guilherme Kraemer de Aguiar

\begin{abstract}
Resumo
A obesidade é uma doença crônica cuja prevalência vem aumentando consideravelmente, tornando-se um grave problema de saúde pública. Na atualidade, um dos principais motivos para o aumento em sua prevalência está relacionado às mudanças nos hábitos alimentares com privilégio para um maior consumo de refeições ricas em gorduras, principalmente as saturadas. Além disso, essa ingestão excessiva de gorduras tem ocorrido em intervalos cada vez menores entre as refeições, o que resulta em um aumento exagerado e prolongado dos níveis de lipoproteínas plasmáticas no período pós-prandial. Tal fato gera o prolongamento e a exacerbação do estado de lipemia pós-prandial (LPP). A LPP tem sido apontada como fator de risco para doenças cardiovasculares (DCV). Em obesos, as consequências da LPP são agravadas pelo excesso de gordura visceral, a qual se relaciona com resistência insulínica (RI) e hiperinsulinemia. Estas alterações da homeostase glicêmica associadas à LPP alteram a função vascular e podem promover disfunção endotelial não só em períodos de jejum, mas também no período pós-prandial. A disfunção endotelial é considerada um marcador precoce para o processo aterosclerótico capaz de elevar o risco de eventos cardiovasculares, como o infarto agudo do miocárdio. As incretinas são secretadas durante a digestão dos alimentos por células específicas localizadas no intestino. Sua principal função está relacionada ao controle da saciedade e a sua ação positiva na secreção de insulina. Entretanto, estudos recentes demonstram que as incretinas, em especial o peptídeo-1 glucagon-símile (GLP-1), podem exercer também efeitos benéficos sobre o sistema vascular. Sua ação pode estar reduzida em diabéticos do tipo 2 e esta redução está intrinsecamente relacionada com a fisiopatologia desta doença. Em diabéticos do tipo 2, a redução dos níveis de GLP-1 pode determinar um prejuízo à saciedade, à secreção de insulina glicose-dependente e ainda associar-se ao aumento do risco de DCV. Em obesos sugere-se que esses efeitos seriam similares àqueles observados em pacientes com diabetes do tipo 2, e talvez atenuados. Assim, esta revisão tem o objetivo de mostrar o papel das incretinas na modulação lipídica durante a LPP e sua relação com a reatividade endotelial, com foco em estudos em indivíduos obesos.
\end{abstract}

Descritores: Obesidade; Endotélio; Incretinas.

\begin{abstract}
Obesity is a chronic disease whose prevalence has been increasing considerably, becoming a serious public health problem. Currently, the increase in its prevalence is related to the changes in eating habits, which indicates higher intake of high-fat meals, mainly saturated fat. Moreover, such excessive consumption has happened in shorter intervals between meals and it causes a prolonged state of postprandial lipemia, currently considered one of the main factors for metabolic abnormalities, such as insulin resistance (IR) and cardiovascular disease (CVD). In this process, obese subjects are considered more problematic, because the excessive amount of visceral fat reduces insulin sensitivity and causes hyperinsulinemia, altering the homeostasis of the vascular system, with consequent microvascular and endothelial dysfunction, being the latter a risk factor for the onset of early atherosclerosis and acute events such as acute myocardial infarction (AMI). Recent studies have shown that incretins, specifically the glucagon-like peptide-1 (GLP-1), may exert beneficial effects on the cardiovascular system. It is also suggested that in obesity and type 2 diabetes mellitus, incretins present reduced action, increasing CVD risk and affecting the satiety control. This review aims to clarify the importance of incretins in modulating lipids during postprandial lipemia and its relation to microvascular dysfunction, focusing on studies performed in obese subjects.
\end{abstract}

Keywords: Obesity; Endothelium; Incretins. 


\section{Introdução}

A obesidade é uma doença crônica definida pelo excesso de gordura corporal, sendo resultado de um desequilíbrio energético influenciado por fatores comportamentais, ambientais e genéticos. ${ }^{1}$ Atualmente, a obesidade é considerada um problema de saúde pública em todo o mundo por aumentar a prevalência de doenças cardiovasculares, resistência à insulina (RI) e diabetes mellitus do tipo 2 (DM2). ${ }^{2}$

Nos dias atuais, o consumo excessivo de gorduras resulta no aumento exagerado e prolongado dos níveis de triglicerídeos (TG) no período pós-prandial, considerado um fator de risco cardiovascular. Esse estado de hipertrigliceridemia pós-prandial, também conhecido com lipemia pós-prandial (LPP), até o momento, não tem uma definição amplamente aceita e padronizada. A LPP per se está associada ao aumento do risco cardiovascular. ${ }^{3}$

Durante a digestão de alimentos, há a produção de peptídeos com ação incretínica pelas células enteroendócrinas do íleo distal, denominados incretinas. Estas, por sua vez, ao serem liberadas na circulação, estimulam a secreção de insulina pelas células beta pancreáticas, retardam o esvaziamento gástrico e reduzem o apetite. Além disso, são capazes de reduzir a glicemia através da inibição da secreção de glucagon pelas células alfa pancreáticas. Porém, muitos estudos têm demonstrado que estas incretinas - em especial o peptídeo-1 glucagon-símile (GLP-1) e o polipeptídeo insulinotrópico glicose-dependente (GIP) -, também seriam importantes reguladores da reatividade vascular ${ }^{4}$ e seu papel na patogênese da aterosclerose ainda permanece desconhecido. Com isso, o objetivo desta revisão é, de acordo com o que existe na literatura, mostrar a possível relação entre a LPP, a disfunção endotelial e as incretinas.

\section{O endotélio saudável e patológico}

Em condições fisiológicas, o endotélio mantém a homeostase vascular, regulando o tônus vascular pelo equilíbrio entre a produção de moléculas vasodilatadoras, como o óxido nítrico (NO), a prostaciclina, o fator hiperpolarizante derivado do endotélio (Endothelium Derived Hyperpolarizing
Factor - EDHF) e vasoconstritoras, como a endotelina-1 e a angiotensina, entre outros agentes pró -aterogênicos. Estes mediadores da reatividade vascular agem diretamente na fluidez do sangue e também na coagulação, através da produção de fatores que modulam a atividade plaquetária, a cascata de coagulação e a fibrinólise. ${ }^{5}$ A ativação endotelial ocorre quando há uma desregulação desses mediadores com predomínio dos fatores pró-aterogênicos, que resulta em um estado denominado disfunção endotelial. ${ }^{6}$ Este é o primeiro marcador de aterosclerose, que pode ser medido clínica e laboratorialmente e, na permanência de fatores clínicos de risco pró-aterogênicos, esse processo poderá promover o desenvolvimento de aterosclerose na parede do vaso sanguíneo acometido.

Na maioria dos casos, o dano ao endotélio que ocorre em indivíduos obesos, fumantes, diabéticos, resistentes à insulina, hipertensos ou com doenças inflamatórias é ocasionado pelo aumento do estresse oxidativo, dos níveis circulantes dos ácidos graxos livres (AGLs) e seus metabólitos, e de moléculas pró-inflamatórias. ${ }^{6,7}$

Na obesidade, a disfunção endotelial pode ser desencadeada através do acúmulo dos radicais livres secundários ao excesso da oxidação lipídica mitocondrial ou ao estímulo de sua produção através de mediadores de inflamação de baixo grau, provenientes de adipócitos, principalmente localizados na gordura visceral. A pletora de substratos lipídicos pode ocorrer secundariamente ao consumo excessivo e consecutivo de refeições ricas em gordura. ${ }^{8}$

\section{Microcirculação e obesidade}

Os vasos que possuem diâmetros inferiores a $100 \mu \mathrm{m}$, incluindo arteríolas, capilares e vênulas, ${ }^{9}$ são considerados componentes da microcirculação e têm como principal função o aporte de nutrientes e oxigênio aos tecidos e a remoção de excretas do metabolismo celular, em resposta às variações da demanda de cada órgão. ${ }^{10}$ Além disso, outra função não menos importante é a manutenção da pressão hidrostática e consequentemente o controle da resistência vascular periférica através de arteríolas de pequeno calibre. ${ }^{9}$

Há muito se pensava que somente os portadores de DM2 teriam alterações microcirculató- 
rias. Entretanto, com o advento de técnicas de avaliação da reatividade microvascular capazes de detectar alterações muito precoces na reatividade desses microvasos, foi possível observar que adultos obesos com ou sem o diagnóstico de síndrome metabólica, porém normoglicêmicos e normotensos, já apresentavam disfunção microvascular. ${ }^{11-13}$ Mesmo em adolescentes com obesidade, a ocorrência de disfunção microvascular foi observada. ${ }^{14}$ Tal fato chamou a atenção para a obesidade como fator preditor de doença microvascular e sugeriu que estas alterações têm início muito precocemente, estão relacionadas à obesidade per se e podem apresentar evolução temporal no espectro de indivíduos portadores de excesso de peso. Consequentemente, como a evolução habitual dos indivíduos portadores de obesidade é o aparecimento de comorbidades, como o DM2 e a hipertensão arterial, pode-se esperar que no diagnóstico destas, a disfunção microvascular já esteja presente e seja possivelmente de longa evolução.

\section{Incretinas e sistema circulatório}

Alguns minutos após a refeição, as incretinas são liberadas na circulação através das células enteroendócrinas. Estes peptídeos ligam-se às proteínas $\mathrm{G}$ presentes nas células beta pancreáticas e ainda em outros tecidos-alvo. ${ }^{15}$ Sua ação no pâncreas permite um incremento na secreção insulínica glicose-dependente por estimulação da síntese de insulina e ainda a inibição da secreção do glucagon nas células alfa. Além de serem importantes na homeostase de glicose, seu papel no esvaziamento gástrico e na regulação do apetite, com consequente redução da ingesta alimentar, ${ }^{16}$ também já foi comprovado.

O GLP-1 é secretado em maiores concentrações do que o GIP e fisiologicamente tem maior relevância em humanos, apresentando como importantes funções, além das citadas anteriormente, a redução do fluxo da linfa intestinal, da absorção de triglicerídeos e da síntese de apolipoproteínas. Tal cenário pode limitar a liberação de triglicerídeos na circulação e consequentemente reduzir a amplitude dessa resposta durante a LPP. $^{17}$

Portadores de obesidade, principalmente aqueles com DM2 associado, têm níveis reduzidos de incretinas. ${ }^{16,18}$ Tal fato é fator causal de piora na homeostase glicêmica e, atualmente, para o controle de pacientes com DM2, empregam-se drogas incretinomiméticas, com base nesta alteração fisiopatológica.

Estudos recentes têm demonstrado a importância do receptor específico do GLP-1 (GLP-1R) em humanos, que podem ser encontrados nas células endoteliais da coronária e também na veia umbilical, ${ }^{19,20} \mathrm{e}$ em animais, nas células endoteliais da coronária e também nas células musculares lisas do sistema vascular. ${ }^{21}$ Nas células beta pancreáticas, a ativação do GLP-1R aumenta os níveis intracelulares de AMP cíclico, estimulando a secreção de insulina dependente de glicose. ${ }^{22}$ Já no endotélio, a ativação do GLP-1R estimula a produção de NO, promovendo relaxamento do músculo liso vascular e melhora da resposta vasodilatadora, uma ação semelhante a da insulina ao ligar-se ao seu receptor, localizado nas células endoteliais. Consequentemente, em estados fisiológicos, a ativação do GLP-1R através de sua ligação com o GLP-1, produzido durante a digestão dos alimentos, promove não só ações relacionadas à digestão, mas também ações hemodinâmicas e parece ser importante na indução de um efeito protetor ao endotélio. ${ }^{21,23-26}$

Além disso, tem havido um grande interesse da ação do GLP-1 no coração. Estudos recentes em modelos in vivo de isquemia miocárdica em ratos têm relacionado a redução da área isquêmica após a infusão do GLP-1. ${ }^{27}$ Estudos clínicos corroboraram as observações anteriores e demonstraram que o GLP-1 induz à melhora da função do miocárdio após infarto agudo do miocárdio ${ }^{28}$ em portadores de DM2 e insuficiência cardíaca congestiva. $^{29}$

\section{Lipemia pós-prandial, obesidade e risco cardiovascular}

Desde 1979, quando Zilversmit trabalhou com a hipótese de que o desenvolvimento da aterosclerose seria um fenômeno pós-prandial, ${ }^{30}$ sugeriu-se que a LPP poderia ser um fator de risco para DCV, principalmente em indivíduos com desordens metabólicas, como obesidade e DM2. ${ }^{31} \mathrm{O}$ dano ao endotélio seria secundário ao aumento exagerado e prolongado dos níveis 
dos TG plasmáticos no período pós-prandial por meio dos mecanismos descritos anteriormente. Apesar de ainda ser objeto de questionamentos, ${ }^{32}$ há evidências de que obesos, após a ingestão de uma refeição rica em lipídios, apresentam uma elevação exagerada e prolongada dos níveis de TG, de glicose, de insulina e dos AGLs quando comparados àqueles hígidos. ${ }^{31,33}$

Cabe ressaltar que a LPP é um fenômeno fisiológico, mas esta homeostase pode ser desfeita em indivíduos com obesidade. O exagero na resposta dos nutrientes e hormônios já mencionados determinaria a estes indivíduos maior risco de dano vascular. Por este motivo, as alterações pós-prandiais no metabolismo de TG e lipoproteínas, com resultante acúmulo desses lipídeos na circulação, são consideradas atualmente fatores fundamentais para a deflagração e perpetuação do processo aterosclerótico. ${ }^{34}$

\section{Relação entre disfunção endotelial/ microvascular, incretinas e estado pós-prandial}

Poucos estudos avaliaram a função endotelial no estado pós-prandial na população obesa. $\mathrm{Na}$ tabela 1 é possível observar que há uma grande heterogeneidade na metodologia empregada nestes estudos. Recentemente, Jonk e colaboradores observaram que após a ingestão de uma refeição mista, ou seja, contendo carboidratos, proteínas e lipídeos em proporções recomendadas, indivíduos com obesidade apresentavam redução da vasodilatação endotélio-dependente na microcirculação cutânea quando comparados com indivíduos saudáveis. ${ }^{35}$ Os autores sugeriram que a hiperglicemia encontrada em obesos no período pós-prandial seria o principal estímulo para a produção de espécies reativas de oxigênio e dano vascular. Além disso, estes autores ressaltaram que a hipertrigliceridemia causada pela ingestão da refeição mista empregada no estudo poderia acentuar a resistência à insulina e prejudicar a função endotelial dos obesos. Em contraposição a este achado, Ayer e colaboradores, ao testar a reatividade micro e macrovascular durante a LPP, observaram respostas similares entre obesos e controles após a ingestão da refeição hiperlipídica. ${ }^{32}$
Em 2009, através de técnicas desenvolvidas recentemente capazes de mensurar a reatividade microvascular diretamente no tecido muscular, Keske e colaboradores observaram a ocorrência de disfunção microvascular em obesos após a ingestão de uma refeição mista. ${ }^{36}$ Esses autores sugeriram que a provável explicação para esse achado seria a ocorrência de hiperinsulinemia exacerbada em decorrência da resistência insulínica no grupo obeso. Sabe-se que em estado de repouso somente um terço dos capilares do tecido muscular são perfundidos. Entretanto, na dependência da demanda (contração muscular/ aumento da insulinemia), haverá um incremento no número de capilares perfundidos para permitir a manutenção da homeostase tecidual através de maior área de perfusão microvascular. Este incremento, denominado recrutamento capilar, pode ser testado com técnicas de reatividade microvascular e guarda estreita relação com a homeostase glicêmica. ${ }^{11,37} \mathrm{Em}$ 2013, Van Genugten e colaboradores, ${ }^{33}$ corroboram os achados de Keske e colaboradores ${ }^{36}$ no tecido muscular. Nesses estudos, eles avaliaram a função microvascular após uma refeição hiperlipídica em três grupos de pacientes: DM2, com síndrome metabólica e controles saudáveis, e constataram que durante a LPP os controles apresentaram incremento do recrutamento capilar, ao contrário dos portadores de síndrome metabólica e de DM2. A redução no recrutamento capilar durante a LPP nos diabéticos e também naqueles com síndrome metabólica esteve relacionada com a redução da sensibilidade à insulina e também com a hiperglicemia pós-prandial. Tal fato sugere que o defeito de perfusão microvascular, também denominado defeito pré-receptor na ação insulínica, influenciaria a homeostase glicêmica no estado pós-prandial.

Sabe-se que através da via do $\mathrm{NO}^{24}$ e também por redução do estresse oxidativo, ${ }^{19}$ a administração endovenosa do GLP-1 promove aumento na vasodilatação endotélio-dependente em indivíduos com DM2. ${ }^{38}$ Consequentemente, é possível que, durante a refeição, esse peptídeo possa exercer simultaneamente um efeito incretínico e também vasculoprotetor. Entretanto, estudos que comprovem ou contradigam esta hipótese ainda são inexistentes.

Um estudo publicado recentemente demonstrou que, ao suprimir o GLP-1, a hiperglicemia 
secundária a esta supressão causaria estresse oxidativo e, consequentemente, disfunção endotelial. Este efeito foi bloqueado ou diminuído na presença desse peptídeo. ${ }^{38}$ Grande parte das investigações com administração de agonistas do GLP-1 em humanos demonstraram sua eficiência na redução dos níveis de TG, dos AGLs e da glicemia pós-prandial. ${ }^{39}$ Estudos que inter-relacionam as funções endotelial e microvascular com as incretinas em portadores de obesidade e de DM2 ainda não foram realizados e atualmente são objetos de estudo desse laboratório.

\section{Conclusão}

Esta revisão objetivou apresentar resultados recentes de pesquisas clínicas já publicadas e que demonstram a possível ação das incretinas em modular a lipemia pós-prandial e a função vascular em indivíduos obesos. É possível observar que ainda há poucos trabalhos na literatura com foco nesta inter-relação.

Além disso, esta revisão aponta para a impor- tância do efeito protetor das incretinas na função endotelial e para a necessidade do desenvolvimento de mais estudos em indivíduos com desordens metabólicas.

\section{Referências}

1. Grundy SM. Multifactorial causation of obesity: implications for prevention. Am J Clin Nutr. 1998 Mar;67(3 Suppl):563S-72S.

2. Maron DJ, Fair JM, Haskell WL. Saturated fat intake and insulin resistance in men with coronary artery disease. The Stanford Coronary Risk Intervention Project Investigators and Staff. Circulation. 1991 Nov;84(5):2020-7. http://dx.doi.org/10.1161/01. CIR.84.5.2020

3. Kolovou GD, Mikhailidis DP, Nordestgaard BG, Bilianou H, Panotopoulos G. Definition of postprandial lipaemia. Curr Vasc Pharmacol. 2011 May;9(3):292-301.

4. Inzucchi SE, McGuire DK. New drugs for the treatment of diabetes: part II: Incretin-based therapy and beyond. Circulation. 2008 Jan 29;117(4):57484. http://dx.doi.org/10.1161/CIRCULATIONA-

Tabela 1. Comparação dos principais estudos avaliando o estado pós-prandial em obesos.

\begin{tabular}{|c|c|c|c|c|c|}
\hline Autores & População & $\begin{array}{l}\text { Quantidade } \\
\text { de gordura } \\
\text { (em g) }\end{array}$ & $\begin{array}{l}\text { Duração e } \\
\text { tempos de coleta } \\
\text { (em min) }\end{array}$ & $\begin{array}{l}\text { Métodos de inves- } \\
\text { tigação vascular } \\
\text { empregados }\end{array}$ & Achados \\
\hline $\begin{array}{l}\text { Van Genugten } \\
\text { et al. (2013) }\end{array}$ & $\begin{array}{l}\text { Homens com } \\
\operatorname{DM} 2(n=12) \text { vs. } \\
\text { SM }(n=13) \text { vs. } \\
\text { Controles }(n=12)\end{array}$ & 50 & $\begin{array}{c}0,60,120 \\
180,240\end{array}$ & Videocapilaroscopia & $\begin{array}{l}\text { O recrutamento capilar apresen- } \\
\text { tou-se prejudicado em indivídu- } \\
\text { os com síndrome metabólica } \\
\text { e diabéticos ao contrário dos } \\
\text { controles. }\end{array}$ \\
\hline $\begin{array}{l}\text { Jonk et al. } \\
\text { (2011) }\end{array}$ & $\begin{array}{l}\text { Obesos ( } n=19) \\
\text { vs. Controles } \\
\text { ( } n=20) \text { (ambos os } \\
\text { sexos) }\end{array}$ & 8,3 & $0,75,105$ & $\begin{array}{c}\text { Videocapilarosco- } \\
\text { pia; LDF }\end{array}$ & $\begin{array}{l}\text { Comparando com os controles, } \\
\text { os obesos apresentam a função } \\
\text { endotelial prejudicada no estado } \\
\text { pós-prandial. }\end{array}$ \\
\hline $\begin{array}{l}\text { Ayer et al. } \\
(2010)\end{array}$ & $\begin{array}{l}\text { Obesos ( } n=11) \\
\text { vs. Controles } \\
\text { ( } n=11) \text { (ambos os } \\
\text { sexos) }\end{array}$ & 60 & $0,60,180$ & $\begin{array}{l}\text { RH-PAT; PWV; } \\
\text { FBF; FMD }\end{array}$ & $\begin{array}{l}\text { A resposta vascular a uma refeição } \\
\text { rica em lipídeos é similar em obe- } \\
\text { sos e controles. }\end{array}$ \\
\hline $\begin{array}{c}\text { Keske et al. } \\
\text { (2009) }\end{array}$ & $\begin{array}{l}\text { Controles }(n=8) \\
\text { vs. Obesos }(n=8) \\
\text { (ambos os sexos) }\end{array}$ & $8^{*}$ & $0,60,120$ & CEU e Doppler & $\begin{array}{l}\text { Após a refeição mista, o recru- } \\
\text { tamento capilar ocorre nos con- } \\
\text { troles, mas está diminuído em } \\
\text { obesos. }\end{array}$ \\
\hline $\begin{array}{l}\text { Gill et al. } \\
(2004)\end{array}$ & $\begin{array}{l}\text { Homens obesos } \\
\text { ( } n=10) \text { vs. Con- } \\
\text { troles }(n=10)\end{array}$ & 80 & $\begin{array}{c}0,120,240 \\
360,480\end{array}$ & $\begin{array}{c}\text { Iontoforese e } \\
\text { exercício }\end{array}$ & $\begin{array}{l}\text { O exercício físico melhora a } \\
\text { função vascular em jejum e na } \\
\text { LPP em homens de meia idade. }\end{array}$ \\
\hline
\end{tabular}

LDF - laser-doppler fluxometria; FMD - dilatação fluxo-mediada; FBF - fluxo de sangue do antebraço; PWV - velocidade de onda de pulso da carótida; RH-PAT - tonometria arterial; CEU - ultrassom amplificado por contraste; SM - síndrome metabólica.

* refeição mista com $8 \mathrm{~g}$ de gordura. 


\section{HA.107.735795}

5. Lüscher TF, Barton M. Biology of the endothelium. Clin Cardiol. 1997 Nov;20(11 Suppl 2):Il-3-10.

6. Hamilton SJ, Chew GT, Watts GF. Therapeutic regulation of endothelial dysfunction in type 2 diabetes mellitus. Diab Vasc Dis Res. 2007 Jun;4(2):89-102. http://dx.doi.org/10.3132/dvdr.2007.026

7. Widlansky ME, Gokce N, Keaney JF Jr., Vita JA. The clinical implications of endothelial dysfunction. J Am Coll Cardiol. 2003 Oct 1;42(7):1149-60. http://dx.doi. org/101016/S0735-1097(03)00994-X

8. Beckman JS, Koppenol WH. Nitric oxide, superoxide, and peroxynitrite: the good, the bad, and ugly. Am J Physiol. 1996 Nov;271(5 Pt 1):C1424-37.

9. Levy BI, Ambrosio G, Pries AR, Struijker-Boudier HA. Microcirculation in hypertension: a new target for treatment? Circulation. 2001 Aug 7;104(6):735-40. http://dx.doi.org/10.1161/hc3101.091158

10. Verdant C, De Backer D. How monitoring of the microcirculation may help us at the bedside. Curr Opin Crit Care. 2005 Jun;11(3):240-4.

11. Kraemer-Aguiar LG, Maranhao PA, Sicuro FL, Bouskela E. Microvascular dysfunction: a direct link among BMI, waist circumference and glucose homeostasis in young overweight/obese normoglycemic women? Int J Obes (Lond). 2010 Jan;34(1):111-7. http:// dx.doi.org/10.1038/ijo.2009.209

12. Kraemer-Aguiar LG, Laflor CM, Bouskela E. Skin microcirculatory dysfunction is already present in normoglycemic subjects with metabolic syndrome. Metabolism. 2008 Dec;57(12):1740-6. http://dx.doi. org/10.1016/j.metabol.2008.07.034

13. Kraemer-Aguiar LG, Maranhao PA, Cyrino FZ, Bouskela E. Waist circumference leads to prolonged microvascular reactive hyperemia response in young overweight/obese women. Microvasc Res. 2010 Dec;80(3):427-32. http://dx.doi.org/10.1016/j. mvr.2010.08.004

14. Maranhao PA, Kraemer-Aguiar LG, de Oliveira CL, Kuschnir MC, Vieira YR, Souza MG, et al. Brazil nuts intake improves lipid profile, oxidative stress and microvascular function in obese adolescents: a randomized controlled trial. Nutr Metab (Lond). 2011;8(1):32. http://dx.doi.org/10.1186/1743-7075-8-32

15. Holst JJ, Vilsboll T, Deacon CF. The incretin system and its role in type 2 diabetes mellitus. Mol Cell Endocrinol. 2009 Jan 15;297(1-2):127-36. http://dx.doi. org/10.1016/j.mce.2008.08.012

16. Drucker DJ. The biology of incretin hormones. Cell Metab. 2006 Mar;3(3):153-65. http://dx.doi. org/10.1016/j.cmet.2006.01.004

17. Qin X, Shen H, Liu M, Yang Q, Zheng S, Sabo M, et al. GLP-1 reduces intestinal lymph flow, triglycer- ide absorption, and apolipoprotein production in rats. Am J Physiol Gastrointest Liver Physiol. 2005 May;288(5):G943-9. http://dx.doi.org/10.1152/ajpgi.00303.2004

18. Wikarek T, Chudek J, Owczarek A, Olszanecka-Glinianowicz M. Effect of dietary macronutrients on postprandial incretin hormone release and satiety in obese and normal-weight women. Br J Nutr. 2013 Aug 6:1-11. http://dx.doi.org/10.1017/S0007114513002389

19. Nystrom T, Gutniak MK, Zhang Q, Zhang F, Holst JJ, Ahren B, et al. Effects of glucagon-like peptide-1 on endothelial function in type 2 diabetes patients with stable coronary artery disease. Am J Physiol Endocrinol Metab. 2004 Dec;287(6):E1209-15. http:// dx.doi.org/10.1152/ajpendo.00237.2004

20. Ishibashi Y, Matsui T, Takeuchi M, Yamagishi S. Glucagon-like peptide-1 (GLP-1) inhibits advanced glycation end product (AGE)-induced up-regulation of VCAM-1 mRNA levels in endothelial cells by suppressing AGE receptor (RAGE) expression. Biochem Biophys Res Commun. 2010 Jan 15;391(3):1405-8. http://dx.doi.org/10.1016/j.bbrc.2009.12.075

21. Ban K, Noyan-Ashraf MH, Hoefer J, Bolz SS, Drucker DJ, Husain M. Cardioprotective and vasodilatory actions of glucagon-like peptide 1 receptor are mediated through both glucagon-like peptide 1 receptor-dependent and -independent pathways. Circulation. 2008 May 6;117(18):2340-50. http://dx.doi.org/10.1161/ CIRCULATIONAHA.107.739938

22. Yabe D, Seino Y. Two incretin hormones GLP-1 and GIP: comparison of their actions in insulin secretion and beta cell preservation. Prog Biophys Mol Biol. 2011 Nov;107(2):248-56. http://dx.doi.org/10.1016/j. pbiomolbio.2011.07.010

23. Golpon HA, Puechner A, Welte T, Wichert PV, Feddersen CO. Vasorelaxant effect of glucagon-like peptide-(7-36)amide and amylin on the pulmonary circulation of the rat. Regul Pept. 2001 Dec 15;102(23):81-6.

24. Basu A, Charkoudian N, Schrage W, Rizza RA, Basu R, Joyner MJ. Beneficial effects of GLP-1 on endothelial function in humans: dampening by glyburide but not by glimepiride. Am J Physiol Endocrinol Metab. 2007 Nov;293(5):E1289-95. http://dx.doi.org/10.1152/ ajpendo.00373.2007

25. Ding L, Zhang J. Glucagon-like peptide-1 activates endothelial nitric oxide synthase in human umbilical vein endothelial cells. Acta Pharmacol Sin. 2012 Jan;33(1):75-81. http://dx.doi.org/10.1038/aps.2011.149

26. Oeseburg H, de Boer RA, Buikema H, van der Harst P, van Gilst WH, Silljé HH. Glucagon-like peptide 1 prevents reactive oxygen species-induced endothelial cell senescence through the activation of protein kinase A. Arterioscler Thromb Vasc Biol. 2010 Jul;30(7):1407-14. http://dx.doi.org/10.1161/ATVBAHA. 110.206425 
27. Bose AK, Mocanu MM, Carr RD, Brand CL, Yellon DM. Glucagon-like peptide 1 can directly protect the heart against ischemia/reperfusion injury. Diabetes. 2005 Jan;54(1):146-51. http://dx.doi.org/10.2337/diabetes.54.1.146

28. Thrainsdottir I, Malmberg K, Olsson A, Gutniak M, Ryden L. Initial experience with GLP-1 treatment on metabolic control and myocardial function in patients with type 2 diabetes mellitus and heart failure. Diab Vasc Dis Res. 2004 May;1(1):40-3. http://dx.doi. org/10.3132/dvdr.2004.005

29. Nikolaidis LA, Mankad S, Sokos GG, Miske G, Shah A, Elahi D, et al. Effects of glucagon-like peptide-1 in patients with acute myocardial infarction and left ventricular dysfunction after successful reperfusion. Circulation. 2004 Mar 2;109(8):962-5. http://dx.doi. org/10.1161/01.CIR.0000120505.91348.58

30. Zilversmit DB. Atherogenesis: a postprandial phenomenon. Circulation. 1979 Sep;60(3):473-85. http:// dx.doi.org/10.1161/01.CIR.60.3.473

31. Gill JM, Al-Mamari A, Ferrell WR, Cleland SJ, Packard CJ, Sattar N, et al. Effects of prior moderate exercise on postprandial metabolism and vascular function in lean and centrally obese men. J Am Coll Cardiol. 2004 Dec 21;44(12):2375-82. http://dx.doi. org/101016/j.jacc.2004.09.035

32. Ayer JG, Harmer JA, Steinbeck K, Celermajer DS. Postprandial vascular reactivity in obese and normal weight young adults. Obesity (Silver Spring). 2010 May;18(5):945-51. http://dx.doi.org/10.1038/ oby. 2009.331

33. van Genugten RE, Serne EH, Heymans MW, van Raalte DH, Diamant M. Postprandial microvascular function deteriorates in parallel with gradual worsening of insulin sensitivity and glucose tolerance in men with the metabolic syndrome or type 2 diabetes.
Diabetologia. 2013 Mar;56(3):583-7. http://dx.doi. org/10.1007/s00125-012-2783-y

34. Chan DC, Pang J, Romic G, Watts GF. Postprandial hypertriglyceridemia and cardiovascular disease: current and future therapies. Curr Atheroscler Rep. 2013 Mar;15(3):309. http://dx.doi.org/10.1007/s11883013-0309-9

35. Jonk AM, Houben AJ, Schaper NC, de Leeuw PW, Serne EH, Smulders YM, et al. Obesity is associated with impaired endothelial function in the postprandial state. Microvasc Res. 2011 Nov;82(3):423-9. http:// dx.doi.org/10.1016/j.mvr.2011.08.00

36. Keske MA, Clerk LH, Price WJ, Jahn LA, Barrett EJ. Obesity blunts microvascular recruitment in human forearm muscle after a mixed meal. Diabetes Care. 2009 Sep;32(9):1672-7. http://dx.doi.org/10.2337/ dc09-0206

37. Buss C, Kraemer-Aguiar LG, Maranhao PA, Marinho C, de Souza MD, Wiernsperger N, et al. Novel findings in the cephalic phase of digestion: a role for microcirculation? Physiol Behav. 2012 Feb 28;105(4):1082-7. http://dx.doi.org/10.1016/j.physbeh.2011.12.004

38. Ceriello A, Esposito K, Testa R, Bonfigli AR, Marra $\mathrm{M}$, Giugliano D. The possible protective role of glucagon-like peptide 1 on endothelium during the meal and evidence for an "endothelial resistance" to glucagon-like peptide 1 in diabetes. Diabetes Care. 2011 Mar;34(3):697-702. http://dx.doi.org/10.2337/ dc10-1949

39. Meier JJ, Gethmann A, Gotze O, Gallwitz B, Holst JJ, Schmidt WE, et al. Glucagon-like peptide 1 abolishes the postprandial rise in triglyceride concentrations and lowers levels of non-esterified fatty acids in humans. Diabetologia. 2006 Mar;49(3):452-8. http:// dx.doi.org/10.1007/s00125-005-0126-y
Recebido:
16/09/2013
Revisado:
26/11/2013.
Aprovado:
04/02/2014. 